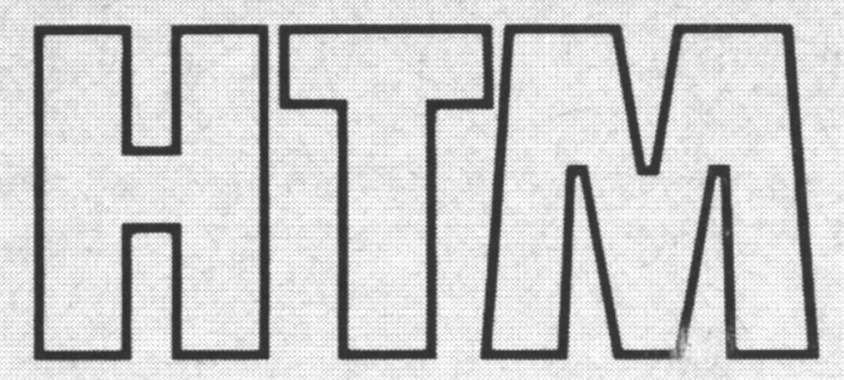

Härterei-Technische Mitteilungen

Vereinigt mit HÄRTEREI-TECHNIK UND WÄRMEBEHANDLUNG

Im Auftrag der Arbeitsgemeinschaft Wärmebehandlung und WerkstoffTechnik e.V. (AWT) herausgegeben von Prof. Dr.-Ing. Otto Schaaber Lesumer Heerstr. 32, Postfach 770207 2820 Bremen 77

und Prof. Dr.-Ing. Walter Stuhlmann

Hochholzweg 14A

7000 Stuttgart-Heumaden

\section{Hauptschriftleitung:}

Prof. Dr.-Ing. Otto Schaaber Stiftung Institut für Härterei-Technik Lesumer Heerstr. 32, Postfach 770207 2820 Bremen 77

In der Schriftleitung arbeiten: Ina Lehnert, Bremen; Erika Sauer, Bremen; Jutta Zeising, Bremen.

\section{Jahrgang/Jahr}

Band 34 (1979) (Gründungsjahr 1941)

\section{Erscheinungsweise}

6 Hefte im Jahr

Manuskripte: sowie Mitteilungen, die den redaktionellen Inhalt betreffen, sind an die Schriftleitung der Härterei-Technischen Mitteilungen, Stiftung Institut für Härterei-Technik, Postfach 770207. 2820 Bremen 77, zu senden.

\section{Carl Hanser Verlag}

Kolbergerstraße 22, D-8000 München 80 Tel. (089) 985861 , Telex 0522837

(c) Copyright by Carl. Hanser Verlag

R. Neményi: Einfluß der dem Randschichthärten vorangehenden Wärmebehandlung auf die Oberflächenhärte

J. Zyśk, J. Tacikowski u. E. Kasprzycka: Die Nitrierbarkeit ausgewählter legierter Stähle

H. Dölle u. V. Hauk: Der theoretische Einfluß mehrachsiger tiefenabhängiger Eigenspannungszustände auf die röntgenographische Span-

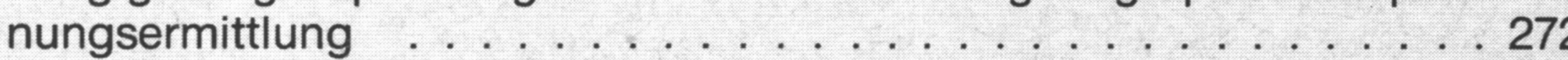

A. Marschall, W. Lode u. A. Peiter: Die Eigenformänderungen oberflächennaher WC-Körner verschiedener Hartmetalle . . . . . . . . . 277

H. Bomas: Das 35. Härterei-Kolloquium 3. bis 5. Oktober 1979 in Wiesbaden

\title{
Dokumentation Wärmebehandlung
}
$A-037-A-052$
Allgemeines
F-005 - F-006
Halbzeugherstellung
G-011 - G-014
Weiterverarbeitung
$\mathrm{J}-206$ - J-252 Technologie der Wärmebehandlung
$\mathrm{K}-011-\mathrm{K}-012$ Fügen
L-059 - L-096 Reinigen, Überzüge, Oberflächenbehandlung
M-115 - M-147 Metallographie, Aufbau und Primärgefüge
$\mathrm{N}-211$ - N-252 Umwandlungen und Umwandlungsgefüge
P-046 - P-053 Physikalische Eigenschaften und Prüfungen
Q-240 - Q-287 Mechanische Eigenschaften und Prüfungen
R-043 - R-047 Korrosion
S-048 - S-053 Prüfung und Überwachung
U-015 - U-024 Verwandte Gebiete
W-076 -W-088 Werkseinrichtungen
X-005 - X-006 Instrumente, Labor- und Überwachungseinrichtungen
AY-08 - TS-06 Werkstoffe 


\section{Verlag und Anzeigenverwaltung Carl Hanser Verlag}

Kolbergerstr. 22, D-8000 München 80 oder Postfach 860420 , München 86

Tel. (089) 985861, Telex 05-22837

Verlagsleiter: Joachim Spencker

Kaufm. Geschäftsführung: F.-J. Klock Herstellungsleiter: Hermann Schlandt Gesamt-Anzeigenleiter: Wolfgang Nagel

Vertriebsleiter: Christoph Sickel

Härtereitechnische Mitteilungen

Herstellung: Irmgard Haase

Anzeigenleiter: Egil Reeg

Inlands-Bezugspreis jährlich DM 177,zuzüglich DM 8,40 Porto (einschließlich $6,5 \%$ MWST). Vorzugspreis für persönliche Mitglieder der AWT jährlich DM 136,50 zuzüglich DM 7,20 Porto. Auslandsbezugspreis jährlich DM 185,40 einschließlich Versandkosten.

Es erscheint jeden zweiten Monat $1 \mathrm{Heft}$. Abbestelltermin spätestens 6 Wochen vor Jahresschluß. Bestellungen nehmen der Buchhandel, die Post oder der Verlag entgegen. Für Interessenten stehen kostenlose Probehefte zur Verfügung.

\section{Härterei-Technische Mitteilungen}

Die in der Zeitschrift veröffentlichten Beiträge sind urheberrechtlich geschützt. Alle Rechte, insbesondere das der Übersetzung in fremde Sprachen, vorbehalten.

Kein Teil dieser Zeitschrift darf ohne schriftliche Genehmigung des Verlages in irgendeiner Form - durch Fotokopie. Mikrofilm oder andere Verfahren - reproduziert oder in eine von Maschinen, insbesondere von Datenverarbeitungsanlagen, verwendbare Sprache übertragen werden.

Fotokopien für den persönlichen und sonstigen eignen Gebrauch, dürfen nur von einzelnen Beiträgen oder Teilen daraus als Einzelkopien hergestellt werden. Jede im Bereich eines gewerblichen Unternehmens hergestellte oder benützte Kopie dient gewerblichen Zwecken gem $\S 54$ (2) UrhG und verpflichtet zur Gebührenzahlung an die VG Wort, vereinigt mit der VG Wissenschaft, Goethestr. 49, 8000 München 2, von der die einzelnen Zahlungsmodalitäten zu erfragen sind Die Weitergabe von Vervielfältigungen gleichgültig zu welchem Zweck sie hergestellt werden, ist eine Urheberrechtsverletzung.

Durch den Verlag können Fotokopien zum Preis von DM 1,60 je DIN-A 4-Seite bezogen werden.

Die Wiedergabe von Gebrauchsnamen, Handelsnamen, Warenbezeichnungen und dgl. in dieser Zeitschrift berechtigt nicht zu der Annahme, daß solche $\mathrm{Na}$ men ohne weiteres von jedermann benutzt werden dürfen; oft handelt es sich um gesetzlich geschützte eingetragene Warenzeichen, auch wenn sie nicht als solche gekennzeichnet sind.

(C) Copies of articles may be made for personal or internal use, or for the use of specific clients. This consent is given on the condition, however, that the copier pay US $\$ 0.25$ fee per page through the Copyright Clearance Center, Inc., P.O. BOX 765, Schenectady, New York 12301, for copying beyond that permitted by Sections 107 or 108 of the U.S. Copyright Law.

Arbeitsgebiet:

Die Härterei-Technischen Mitteilungen vermitteln mit ihren Originalarbeiten inund ausländischer Autoren einen umfassenden Überblick über die mit den Grundlagen und der Technologie der Wärmebehandlung zusammenhängenden Fragenkomplexe. Diese Berichte über neue bei Forschungsarbeiten gewonnene Erkenntnisse und über Erfahrungen mit Werkstoffen, Verfahren und Einrichtungen in der betrieblichen Wärmebehandlung werden im Schrifttumsteil durch einen Dokumentationsdienst ergänzt, der die ganze Weltliteratur über Wärmebehandlung und damit verbundene Gebiete erfaßt.

In den Härterei-Technischen Mitteilungen erscheinen u. a. die Vorträge des Härterei-Kolloquiums sowie ausgewählte Beiträge der internationalen Veranstaltungen über Wärmebehandlungsfragen. Das Institut für Härterei-Technik, Bremen, veröffentlicht die Ergebnisse seiner Forschungsarbeiten auf dem Gebiet der Wärmebehandlung in den Härterei-Technischen Mitteilungen.

Anzeigen-Grundpreise:

$1 / 1$ Seite 1060,-DM

$250 \mathrm{~mm}$ hoch

$176 \mathrm{~mm}$ breit

$1 / 2$ Seite $530,-D M$

$122 \mathrm{~mm}$ hoch

$176 \mathrm{~mm}$ breit

oder $250 \mathrm{~mm}$ hoch

$85 \mathrm{~mm}$ breit

$1 / 4$ Seite $265-D M$

$22 \mathrm{~mm}$ hoch $85 \mathrm{~mm}$ breit

oder $58 \mathrm{~mm}$ hoch

$176 \mathrm{~mm}$ breit

$1 / 8$ Seite $133,-D M$

$58 \mathrm{~mm}$ hoch $85 \mathrm{~mm}$ breit

oder $28 \mathrm{~mm}$ hoch

$176 \mathrm{~mm}$ breit

$1 / 16$ Seite $67,-$ DM $\quad 58 \mathrm{~mm}$ hoch

$41 \mathrm{~mm}$ breit

oder $28 \mathrm{~mm}$ hoch

$85 \mathrm{~mm}$ breit

Grundpreis für die einspaltige $\mathrm{mm}$-Zeile $1,25 \mathrm{DM}$

\section{Generalvertretungen des Carl Hanser Verlags}

\section{Inland}

Berlin

Hans-C. Reisner, Carstennstr. $61 \mathrm{~A}$

D-1000 Berlin 45, Tel. 030/8174077

\section{Coburg}

Oskar Ohler, Stockäckerstr. 6

D-8630 Coburg 12, Postfach 622

Tel. 09561/66122

Telex: 06-63317

\section{Frankfurt/M.}

Dr. Karl Niedermeyer Nachf.

Georg-Speyer-Str. 76

D-6000 Frankfurt/Main 90

Tel. 0611/775036

Hagen

W. O. Hirnschal, Annaberghöhe 2

D-5800 Hagen i. W., Tel. 02331/53252

\section{Ausland}

Frankreich

Publicitas ANC RIPSA

26, Avenue Victor-Hugo

F-75116 Paris

Tel. 5006608

Telex 610067

\section{Großbritannien}

Bondway Publishing Co. Ltd.

6 Cobbett Close, Pound Hill

Crawley RH 10 3DR, Sussex.

Tel. $029382 / 2817+3565$

\section{Holland/Belgien}

A. G. Contant, Aleidastraat 23

Hengelo (Ov.)-Holland

Tel. 05400/18328

\section{Italien}

Cesare Epis, Pubblitecna, S.R.L.

Via Washington, 27

I-20146 Milano

Tel.: 482941 u. 482942

\author{
Hamburg \\ Jörg Fockele \\ Rütestr. 92, D-2000 Hamburg 70 \\ Tel.: 040/685915 und 612569
}

\section{Hannover}

Hagen Döhner, Hegelstr. 10

D-3000 Hannover-Kleefeld, Postfach 364 Tel. 0511/552325

\section{Stuttgart}

P. Scholl, Verlagsvertretungen

Wiflingshauser Str. 83

D-7300 Esslingen a. $\mathrm{N}$

Tel. 0711/371673, Telex: 07/256406

Wuppertal

H. W. Gerstner

Odenwaldweg 3, D-5600 Wuppertal 12

Tel. 0202/400117

Telex 08/591801

Japan

International Media Representatives Ltd.

1, Shiba-Kotohiracho Minatoku, Tokyo

Cable: mediarep tokyo

Tel.: 502-0656, Telex: J 22633

\section{Ósterreich}

Eva Franklin, Sonnenweg 84

A-1140 Wien, Tel. 9424503

\section{Skandinavien}

Sveadress, P.O. Box 4085

S-12740 Skärholmen 4

Tel. Stockholm 08-7103700

Telex: 13488 sveadre s

\section{Schweiz}

Robert Dormann, Postfach 2066

(Alte Landstr. 197), CH-8800 Thalwil

Tel. 01/72085 50

\section{USA}

INTA Advertising, Inc.

1560 Broadway, New York, N. Y. 10036

Tel. (212) 575-9292, Telex: 422260 